\title{
Pravastatin lowered coronary disease risk in elderly persons with or at risk of vascular disease
}

\author{
Shepherd J, Blauw GJ, Murphy MB, et al. Pravastatin in elderly individuals at risk of vascular disease (PROSPER): a \\ randomised controlled trial. Lancet 2002;360:1623-30.
}

\section{QUESTION: In elderly persons with or at risk of vascular disease, what is the effectiveness and safety of pravastatin?}

\section{Design}

Randomised (allocation concealed*), blinded (clinicians, participants, data collectors, and outcome assessors),* placebo controlled trial with mean follow up of 3.2 years (Prospective Study of Pravastatin in the Elderly at Risk [PROSPER]).

\section{Setting}

Scotland, Ireland, and the Netherlands.

\section{Participants}

5804 participants between $70-82$ years (mean age 75 y, $52 \%$ women) who had a history of vascular disease (coronary, cerebral, or peripheral) or risk factors for vascular disease (eg, smoking, hypertension, or diabetes), a total plasma cholesterol concentration between 4.0 and 9.0 $\mathrm{mmol} / \mathrm{l}$, and a triglyceride concentration $<6.0 \mathrm{mmol} / \mathrm{l}$. Participants with poor cognitive function (Mini-Mental State Examination score $<24$ ) were excluded. Follow up was $100 \%$.

\section{Intervention}

Participants were allocated to pravastatin, $40 \mathrm{mg}$ /day $(\mathrm{n}=2891)$, or placebo $(\mathrm{n}=2913)$.

\section{Main outcome measures}

Composite endpoint of coronary death, nonfatal myocardial infarction (MI), or fatal or nonfatal stroke (primary composite endpoint); composite endpoint of coronary death or nonfatal MI; composite endpoint of fatal or nonfatal stroke; and adverse events.

\section{Main results}

Analysis was by intention to treat. Pravastatin lowered the risk of the primary composite endpoint and the composite endpoint of coronary death or nonfatal MI (table). The pravastatin and placebo groups did not differ for the composite endpoint of fatal or nonfatal stroke, but pravastatin was associated with a greater risk of having a new cancer diagnosis (table).

\section{Conclusion}

In elderly persons with or at risk of vascular disease, pravastatin lowered the risk of coronary disease events.

*See glossary.

\section{COMMENTARY}

With proof that lowering cholesterol concentrations decreases mortality in high risk, middle aged patients, it is appropriate to focus attention on the elderly. Beyond about 75 years of age, serum cholesterol concentrations contribute less to the risk of coronary heart disease than they do between the ages of $55-75$ years, but coronary mortality is higher.

The results of the PROSPER study extend the results of a subgroup analysis of the Heart Protection Study ${ }^{1}$ that showed significant effects of statin therapy on cardiovascular events in older patients. PROSPER failed to confirm decreased stroke rates with statin therapy, probably because of short follow up and a lower than expected background stroke rate. Many participants in the study, however, had systolic hypertension at baseline (mean systolic blood pressure $155 \mathrm{~mm} \mathrm{Hg}$ ), and control of this risk factor might have lessened the effect of statin therapy on stroke (and MI) even more. The finding of increased malignancy rates in patients treated with statins should not be viewed as credible. This finding contradicts a larger body of evidence showing that no such increased risk exists and is more likely the result of chance.

Elderly patients and their caregivers often choose therapies that preserve functional status rather than those that decrease mortality. PROSPER failed to show reductions in cognitive and functional decline, but the measures used in the study were insensitive to change in persons with high levels of function.

On the whole, the results of PROSPER give providers and elderly patients data on which to individualise therapeutic decisions. Elderly patients who are highly functional, have vascular disease or high cholesterol concentrations and 1 other risk factor, and wish to maximise life span will probably choose statin therapy. Similar patients whose only goal is to preserve their current functional level will probably forgo therapy.

Edward Havranek, MD Denver Health Medical Center Denver, Colorado, USA

1 Heart Protection Study Collaborative Group. MRC/BHF Heart Protection Study of cholesterol lowering with simvastatin in 20,536 high-risk individuals: a randomised placebocontrolled trial. Lancet 2002;360:7-22.

Pravastatin v placebo in elderly persons with or at risk of vascular disease at mean 3.2 years $\uparrow$

\begin{tabular}{|c|c|c|c|c|}
\hline Outcomes & Pravastatin & Placebo & RRR $(95 \% \mathrm{Cl})$ & NNT (Cl) \\
\hline Primary composite endpoint $\ddagger$ & $14 \%$ & $16 \%$ & $13 \%(1.8$ to 23$)$ & 47 (25 to 359$)$ \\
\hline \multirow[t]{2}{*}{ Coronary death or nonfatal $\mathrm{Ml}$} & $10 \%$ & $12 \%$ & $17 \%(4$ to 29$)$ & 47 (27 to 199$)$ \\
\hline & & & RRI (CI) & NNH \\
\hline Fatal or nonfatal stroke & $4.7 \%$ & $4.5 \%$ & $4 \%(-18$ to 31$)$ & Not significant \\
\hline New cancer diagnoses & $8.5 \%$ & $6.8 \%$ & $24 \%$ (4 to 48$)$ & 61 (33 to 362 ) \\
\hline
\end{tabular}

$\mathrm{\dagger MI}=$ myocardial infarction. Other abbreviations defined in glossary; RRR, RRI, NNT, NNH, and Cl calculated from data in article.

‡Primary composite endpoint $=$ coronary death, nonfatal $\mathrm{MI}$, or fatal or nonfatal stroke. 